\title{
Identifying targets for antibiotic stewardship interventions through analysis of the antibiotic prescribing process in hospitals - a multicentre observational cohort study
}

Jannicke Slettli Wathne $e^{1,2,3^{*}}$ (D) Brita Skodvin ${ }^{1,2}$, Esmita Charani ${ }^{4}$, Stig Harthug ${ }^{1,2}$, Hege Salvesen Blix ${ }^{5,6}$, Roy M. Nilsen ${ }^{7}$, Lars Kåre Selland Kleppe ${ }^{8}$, Marta Vukovic ${ }^{9}$ and Ingrid Smith ${ }^{10^{*}}$

\begin{abstract}
Background: In order to change antibiotic prescribing behaviour, we need to understand the prescribing process. The aim of this study was to identify targets for antibiotic stewardship interventions in hospitals through analysis of the antibiotic prescribing process from admission to discharge across five groups of infectious diseases.

Methods: We conducted a multi-centre, observational cohort study, including patients with lower respiratory tract infections, exacerbation of chronic obstructive pulmonary disease, skin- and soft tissue infections, urinary tract infections or sepsis, admitted to wards of infectious diseases, pulmonary medicine and gastroenterology at three teaching hospitals in Western Norway. Data was collected over a 5-month period and included antibiotics prescribed and administered during admission, antibiotics prescribed at discharge, length of antibiotic therapy, indication for treatment and discharge diagnoses, estimated glomerular filtration rate (eGFR) on admission, antibiotic allergies, place of initiation of therapy, admittance from an institution, patient demographics and outcome data. Primary outcome measure was antibiotic use throughout the hospital stay, analysed by WHO AWaRe-categories and adherence to guideline. Secondary outcome measures were a) antibiotic prescribing patterns by groups of diagnoses, which were analysed using descriptive statistics and b) non-adherence to the national antibiotic guidelines, analysed using multivariate logistic regression.

(Continued on next page)
\end{abstract}

\footnotetext{
*Correspondence: jannicke.slettli.wathne@sav.no; ismith@who.int

${ }^{1}$ Department of Clinical Science, University of Bergen, Jonas Lies vei 87, 5021 Bergen, Norway

${ }^{10}$ Innovation, Access and Use, Department of Essential Medicines and Health Products, World Health Organization (WHO), Avenue Appia 20, 1211 Geneva 27, Switzerland

Full list of author information is available at the end of the article
}

(c) The Author(s). 2020 Open Access This article is licensed under a Creative Commons Attribution 4.0 International License, which permits use, sharing, adaptation, distribution and reproduction in any medium or format, as long as you give appropriate credit to the original author(s) and the source, provide a link to the Creative Commons licence, and indicate if changes were made. The images or other third party material in this article are included in the article's Creative Commons licence, unless indicated otherwise in a credit line to the material. If material is not included in the article's Creative Commons licence and your intended use is not permitted by statutory regulation or exceeds the permitted use, you will need to obtain permission directly from the copyright holder. To view a copy of this licence, visit http://creativecommons.org/licenses/by/4.0/. The Creative Commons Public Domain Dedication waiver (http://creativecommons.org/publicdomain/zero/1.0/) applies to the data made available in this article, unless otherwise stated in a credit line to the data. 
(Continued from previous page)

Results: Through analysis of 1235 patient admissions, we identified five key targets for antibiotic stewardship interventions in our population of hospital inpatients; 1) adherence to guideline on initiation of treatment, as this increases the use of WHO Access-group antibiotics, 2) antibiotic prescribing in the emergency room (ER), as 83.6\% of antibiotic therapy was initiated there, 3) understanding prescribing for patients admitted from other institutions, as this was significantly associated with non-adherence to guideline (OR=1.44 95\% Cl 1.04, 2.00), 4) understanding cultural and contextual drives of antibiotic prescribing, as non-adherent prescribing differed significantly between the sites of initiation of therapy (between hospitals and ER versus ward) and 5) length of therapy, as days of antibiotic therapy was similar across a wide range of diagnoses and with prolonged therapy after discharge.

Conclusions: Analysing the process of antibiotic prescribing in hospitals with patient-level data identified important targets for antibiotic stewardship interventions in hospitals.

Keywords: Antimicrobial, Stewardship, Antibiotic, Prescribing, Process, AWaRe, Guideline, Hospital, Target, Intervention

\section{Background}

Suboptimal use of antibiotics is a key driver of antibiotic resistance [1]. In order to improve the antibiotic prescribing process, we need to understand it. Historically, antibiotic sales statistics have been easy to collect, and are therefore widely used as a proxy indicator to monitor antibiotic prescribing [2-5]. Although analyses of antibiotic sales data are useful at an aggregated level, they do not specify patient level use or outcomes. Whilst providing a baseline, such data cannot be used to assess the appropriateness of antibiotic prescribing, limiting opportunities for optimising antibiotic stewardship interventions. Accurate, patient level assessment of antibiotic prescribing is an essential step in optimising antibiotic use. Audit and prevalence studies with manual data collection are time-consuming, but often necessary to retrieve this information. Many hospitals still lack electronic medical records that allow automated extraction of antibiotic prescription data with accompanying indications for treatment [6-8]. The introduction of WHO Access, Watch and Reserve (AWaRe) categories have provided a framework for analysing antibiotic consumption, focusing on limiting unnecessary use of watch and reserve antibiotics $[9,10]$. We present the findings of an observational multicentre cohort study aiming to identify targets for antibiotic stewardship interventions by analysing the antibiotic prescribing process from admission to discharge for individual patients.

\section{Methods}

\section{Study design and setting}

This was an observational, multicentre cohort study across the wards of infectious diseases, pulmonary medicine and gastroenterology at three teaching hospitals in Western Norway [11]. The largest hospitals (denoted A and B hereafter) are emergency care, university hospitals with 1100 and 600 beds, respectively, covering most specialities, except transplant surgery. Hospital $C$ is an emergency care, teaching hospital with 160 beds, which is in close collaboration with Hospital A.

\section{Data collection}

The cohort included patients recruited to an antibiotic stewardship intervention study and consisted of adult patients discharged from study wards between the 10th of February and the 11th of July 2014 with a hospital stay $\geq 24 \mathrm{~h}$ and $\leq 21$ days, receiving antibiotics during admission for an indication within guideline recommendations [11, 12]. If a patient was readmitted during the study period, only the first stay was included. Patients with the following indications were included in the analysis: ${ }^{1)}$ lower respiratory tract infections (LRTI) ${ }^{2)}$ exacerbation of Chronic Obstructive Pulmonary Disease $\left(\mathrm{COPD}\right.$ ex ${ }^{3)}$ urinary tract infections (UTI) ${ }^{4)}$ skin- and soft tissue infections (SSTI) and ${ }^{5)}$ sepsis. Patients were excluded if: a) they were admitted to intervention wards in the post-intervention period; and b) comorbidity and patient outcome data were missing.

Data were collected manually from electronic medical records, including admission notes from the emergency room, medical charts, physicians' clinical notes, discharge letters and laboratory test results. Data included patient demographics, indication for antibiotic treatment, antibiotic use throughout the hospital stay, discharge diagnoses, estimated glomerular filtration rate (eGFR) on admission, length of stay, 30-day readmission, in-hospital and 30-day mortality, comorbidity and admittance from institution. Coded data on discharge diagnoses were retrieved from the hospital administrative system.

\section{Outcomes}

The primary outcome measure was antibiotic regimens used throughout the hospital stay, grouped by AWaRecategories and guidelines adherence on initiation of treatment and analysed at initiation of treatment, after 
first modification of regimen, and at discharge. Secondary outcome measures were antibiotic prescribing patterns by groups of diagnoses and non-adherence to the national antibiotic guidelines, analysed as association with study variables.

\section{Patient characteristics and diagnoses}

To assess comorbidity, the Charlson Comorbidity Index (CCI) was calculated based on ICD-10 diagnoses at discharge $[13,14]$. CCI was categorised as CCI equal to 0 , $1,2,3,4$ or $>4$, with zero being no registered comorbidity and $>4$ substantial comorbidity.

The initial working diagnosis, documented in the electronic medical record for prescribed antibiotics, was used as the principal indication. Patients and treatment regimens were grouped by indications according to Supplement 1 , Table 1 . For patients having several diagnoses, all diagnoses were documented and a variable indicating multiple working diagnosis was created. Coauthor BS (Infectious diseases (ID)-physician) assessed patients with multiple working diagnoses and assigned a primary indication for treatment based on the expectation that the treating physicians were likely to choose antibiotic treatment covering the most severe working diagnosis. Accuracy of diagnoses was defined as the percentage of patients for whom the initial indication for antibiotic treatment matched the discharge diagnosis (group level), defined as the infectious disease diagnosis coded or written in free text in the discharge letter.

\section{Antibiotic prescribing}

Antibiotic regimens could include single or multiple antibiotics. Initially prescribed antibiotic regimens were assessed for adherence according to the Norwegian national antibiotic guidelines, as all hospitals included the national guidelines in their local antibiotic policy. Only first-choice empirical regimen for a given indication was regarded adherent. Assessment of adherence was performed using automated syntax in SPSS for Windows (IBM SPSS Statistics, version 24, USA). Indication for treatment was combined with prescribed active substance(s) to generate the adherence variable and adherence was thereafter adjusted manually for patients with kidney failure or antibiotic allergies.

Anti-infectives for systemic use (ATC-group J01), metronidazole tablets (ATC code P01AB01) and vancomycin tablets (A07AA09) were defined as antibiotics in this study. The prescribed antibiotic regimens were assigned to WHO AWaRe categories [9, 15]. For overview of AWaRe categories and included antibiotics, see Supplement 1 , Table 2. Antibiotics belonging only to the "key access" category were included in the "access" category, while antibiotics belonging to "access-watch" and "watch" were included in the watch category. Since the use of antibiotics in the "reserve" category was minimal, the groups of "watch" and "reserve" were combined for analysis. Several antibiotics frequently used in Norway are not included in WHO AWaRe categories. To be able to include these patients in analysis, a modified version of AWaRe categories was prepared (Supplement 1, Table 2). Of the antibiotics not included in the original AWaRe categories, mecillinam, pivmecillinam, metenamin and tobramycin were added to the "access" category and cefuroxime was added to the "watch" category. If an antibiotic regimen contained both access and watch/reserve-group antibiotics, the regimen was classified as watch/reserve.

\section{Modification of antibiotic therapy}

Modifications that prescribing physicians made to the first antibiotic regimen were defined in four categories: escalation, de-escalation, change within same level or unchanged. Day 1 was the day antibiotic therapy was initiated. Patients with regimens in the unchanged category were not included in analysis of time to change. Definitions of modifications are given in Table 1. Assessment of antimicrobial spectrum and categorisation of change were performed and checked by ID-physicians (authors BS and IS, respectively). Examples are given in Supplement 1, Table 3.

\section{Duration of antibiotic therapy}

Duration of antibiotic therapy was measured in days from the first to the last day of therapy and reported as: 1) mean total days of treatment, including prescribed treatment after discharge, 2) mean days of in-hospital antibiotic therapy and 3) mean days of therapy after discharge. When antibiotic treatment continued after discharge, the day of discharge was counted as in-hospital therapy. Information about antibiotic therapy after discharge was retrieved from the discharge letter and also reported as percentage of patients where post-discharge antibiotics were described.

\section{Data analysis}

Descriptive statistics were applied to describe the prescription patterns. To examine which factors were associated with non-adherence, we used univariate and multivariate logistic regression. A targeted selection of factors were evaluated for the multivariate logistic regression model: place of antibiotic therapy initiation, indication for treatment, hospital site, admission from institution, accuracy between indication for treatment and discharge infection diagnosis, sex, age group, comorbidity measured by $\mathrm{CCI}$, multiple working diagnoses, antibiotic allergies and eGFR. Variables that in univariate analysis had a $p$-value of less than 0.2 were included in the final model. Only the first four variables were 
Table 1 Modifications of antibiotic regimens

\begin{tabular}{|c|c|}
\hline Process measures & Definition \\
\hline \multicolumn{2}{|l|}{ Modification of therapy } \\
\hline Escalation & $\begin{array}{l}\text { Change from oral to intravenous (i.v.) antibiotic treatment within the same antibacterial spectrum, } \\
\text { change to more broad-spectrum treatment, adding an antibiotic to a combination. }\end{array}$ \\
\hline De-escalation & $\begin{array}{l}\text { Change from i.v. to oral antibiotic treatment within the same antibacterial spectrum or change to } \\
\text { more narrow-spectrum treatment. }\end{array}$ \\
\hline Change same level & Change to a regimen within the same antibacterial spectrum and form of administration (i.v./oral). \\
\hline Unchanged & $\begin{array}{l}\text { Regimens where first change of therapy was discontinuation of antibiotics, either during admission } \\
\text { or after discharge. }\end{array}$ \\
\hline Time to first modification of $A B$ regimen & $\begin{array}{l}\text { Time to first escalation/de-escalation/change within same antibacterial spectrum and dosage form } \\
\text { (change of active substance(s), i.v. to oral switch, stopping or adding an antibiotic). }\end{array}$ \\
\hline Number of treatment regimens & $\begin{array}{l}\text { The number of treatment regimens from initiation of treatment until antibiotics prescribed at } \\
\text { discharge }\end{array}$ \\
\hline Day of oral antibiotics & The first day that one or more oral antibiotics were given. \\
\hline
\end{tabular}

associated with non-adherence in univariate analysis and included in the final multivariate model. $P$-values below 0.05 were considered statistically significant for all analysis. Stata SE version 15 (Stata Statistical Software, College Station, TX, USA) was used for all statistical analysis, while SPSS for Windows (IBM SPSS Statistics, version 24, USA) was used for assessment of adherence.

\section{Results}

During the study period, 1544 patients with available comorbidity and outcome data met the inclusion criteria. Of these patients, 309 were admitted in the postintervention period at intervention wards and was therefore excluded, leaving 1235 unique patients included in analysis for this study.

\section{Diagnoses and patient characteristics}

The characteristics of the patients are given in Table 2 . The most frequent diagnosis was LRTI (33.4\%), followed by COPD exacerbations (22.7\%), sepsis (20.1\%), SSTI (12.2\%) and UTI (11.7\%) (not shown in tables). In the group of patients with SSTI, $6.0 \%$ of patients were admitted from an institution, compared to $20.7 \%$ for patients with UTI. When investigating accuracy between the groups of indications for empirical antibiotic treatment and discharge infection diagnoses, there was substantial variation with a range from $41.5 \%$ accuracy for patients initially diagnosed with sepsis, to $95.3 \%$ for patients diagnosed with SSTI.

\section{Empirical antibiotic prescribing}

Prescribed antibiotic regimens were adherent to guidelines for $63 \%$ of patients (Table 3). Antibiotics belonging to the WHO AWaRe "Access" category were prescribed as initial regimen for $74 \%$ of patients in total, while the remaining $26.0 \%$ of antibiotic regimens were from the "Watch/Reserve" category. Where initial antibiotic regimens were adherent to guidelines, $89 \%$ of regimens were in the WHO AWaRe access category (Fig. 1). Second regimens included more antibiotics from the watch/ reserve categories and $71 \%$ of regimens were now in the access category. At discharge, $85 \%$ of regimens from the adherent group were in the access category. Where initial antibiotic regimens were non-adherent to guidelines, $49 \%$ of the regimens were in the access category. This increased to $61 \%$ for the second regimen and then again to $74 \%$ of regimens being the access category at discharge.

The majority (83.6\%) of antibiotic prescriptions were initiated in the emergency room, ranging from $64.8 \%$ of prescriptions for UTI to $96.8 \%$ for sepsis (Table 3). Initiating antibiotic therapy at the ward increased the likelihood for non-adherence to guidelines, compared to prescribing in the emergency room, with an odds-ratio (OR) of $1.7,95 \%$ CI $(1.24,2.36)$ (Table 4$)$. When compared to LRTI, all groups of diagnoses were associated with a higher likelihood of non-adherence, ranging from $\mathrm{OR}=1.42$, 95\% CI $(1.03,1.98)$ for COPD ex to $\mathrm{OR}=$ 1.62 , 95\% CI $(1.09,2.41)$ for UTI (all $p<0.05)$. Being admitted to hospital $B$ was associated with reduced OR of non-adherence compared to hospital $\mathrm{A}$, with an $\mathrm{OR}=$ 0.63, 95\% CI $(0.46,0.86), p=0.004$. Patients admitted from an institution had increased risk of receiving nonadherent antibiotic treatment, OR $=1.44,95 \%$ CI (1.04, 2.00), $p=0.029$. Other factors tested were not associated with prescriptions being non-adherent to guidelines.

\section{Modification of antibiotic therapy}

The initial antibiotic regimen was modified during admission for $61.4 \%$ of the patients, and $20.6 \%$ of initial regimens was continued until discharge and then changed (Table 3). For the remaining patients, the initial antibiotic regimen was either stopped $(9.7 \%)$ or continued after discharge (8.3\%). This pattern varied between 
Table 2 Patient characteristics

\begin{tabular}{|c|c|c|c|c|c|c|}
\hline & $\begin{array}{l}\text { LRTI }(n=412) \\
n(\%)\end{array}$ & $\begin{array}{l}\text { COPD ex }(n=280) \\
\text { n (\%) }\end{array}$ & $\begin{array}{l}\text { Sepsis }(n=248) \\
\text { n (\%) }\end{array}$ & $\begin{array}{l}\text { SSTI }(n=150) \\
n(\%)\end{array}$ & $\begin{array}{l}\text { UTI }(n=145) \\
\mathrm{n}(\%)\end{array}$ & $\begin{array}{l}\text { Total }(N=1235) \\
\text { n }(\%)\end{array}$ \\
\hline \multicolumn{7}{|l|}{ Sex } \\
\hline Male & $196(47.6)$ & $149(53.2)$ & $148(59.7)$ & $101(67.3)$ & $62(42.8)$ & $656(53.1)$ \\
\hline Female & $216(52.4)$ & $131(46.8)$ & $100(40.3)$ & $49(32.7)$ & $83(57.2)$ & $579(46.9)$ \\
\hline \multicolumn{7}{|l|}{ Age } \\
\hline$<=45$ & $43(10.4)$ & $2(0.7)$ & $51(20.6)$ & $52(34.7)$ & $16(11.0)$ & $164(13.3)$ \\
\hline $46-65$ & $88(23.4)$ & $70(25.0)$ & $50(20.2)$ & $48(32.0)$ & $21(14.5)$ & $277(22.4)$ \\
\hline $66-85$ & $192(46.6)$ & $179(63.9)$ & $106(42.7)$ & $37(24.7)$ & $71(49.0)$ & $585(47.4)$ \\
\hline$>85$ & 89 (21.6) & $29(10.4)$ & $41(16.4)$ & $13(8.7)$ & $37(25.5)$ & $209(16.9)$ \\
\hline \multicolumn{7}{|c|}{ Charlson Comorbidity Index } \\
\hline $\mathrm{CCl}=0$ & $163(39.6)$ & $8(2.9)$ & $111(44.8)$ & $108(72.0)$ & $72(49.7)$ & $462(37.4)$ \\
\hline $\mathrm{CCl}=1$ & $109(26.5)$ & $178(63.6)$ & $73(29.4)$ & $23(15.3)$ & $37(25.5)$ & $420(34.0)$ \\
\hline $\mathrm{CCl}=2$ & $58(14.1)$ & $47(16.8)$ & $41(16.5)$ & $9(6.0)$ & $21(14.5)$ & $176(14.3)$ \\
\hline $\mathrm{CCl}=3$ & $32(7.8)$ & $24(8.6)$ & $9(3.6)$ & $5(3.3)$ & $9(6.2)$ & $79(6.4)$ \\
\hline $\mathrm{CCl}=4$ & $14(3.4)$ & $18(6.4)$ & $6(2.4)$ & $3(2.0)$ & $1(0.7)$ & $42(3.4)$ \\
\hline $\mathrm{CCl}>4$ & $36(8.7)$ & $5(1.8)$ & $8(3.2)$ & $2(1.3)$ & $5(3.5)$ & $56(4.5)$ \\
\hline \multicolumn{7}{|c|}{ Admitted from institution } \\
\hline No & $341(82.8)$ & $255(91.1)$ & $203(81.9)$ & $141(94.0)$ & $115(79.3)$ & $1055(85.4)$ \\
\hline Yes & $71(17.2)$ & $25(8.9)$ & $45(18.1)$ & $9(6.0)$ & $30(20.7)$ & $180(14.6)$ \\
\hline \multicolumn{7}{|l|}{$A B$ allergies } \\
\hline Yes & $43(10.5)$ & 38 (13.6) & $19(7.7)$ & $13(8.7)$ & $9(6.2)$ & $122(9.9)$ \\
\hline No & 367 (89.3) 1 missing & $242(86.4)$ & $229(92.3)$ & $137(91.3)$ & $136(93.8)$ & 1111 (90.0) 1 missing \\
\hline \multicolumn{7}{|l|}{ eGFR on admission } \\
\hline$>50$ & $308(74.8)$ & $230(82.1)$ & $187(75.4)$ & $129(86.0)$ & $101(69.7)$ & $955(77.3)$ \\
\hline $10-50$ & $103(25.0)$ & 49 (17.5) & $59(23.8)$ & $21(14.0)$ & $43(29.7)$ & $275(22.3)$ \\
\hline$<10$ & $1(0.24)$ & $0(0.0)$ & $2(0.8)$ & $0(0.0)$ & $1(0.7)$ & $4(0.32)$ \\
\hline Dialysis & $0(0.0)$ & $1(0.4)$ & $0(0.0)$ & $0(0.0)$ & $0(0.0)$ & $1(0.08)$ \\
\hline 30-day mortality & $55(13.4)$ & $19(6.8)$ & $22(8.9)$ & $2(1.3)$ & $6(4.1)$ & $104(8.4)$ \\
\hline 30-day readmission & 78 (18.9) & $76(27.1)$ & $39(15.7)$ & $26(17.3)$ & $37(25.5)$ & $256(20.7)$ \\
\hline Mean LOS $(95 \% \mathrm{CI})$ & $7.3(6.8,7.7)$ & $6.8(6.3,7.2)$ & $7.1(6.6,7.6)$ & $6.3(5.6,7.0)$ & $7.0(6.3,7.7)$ & $7.0(6.7,7.2)$ \\
\hline
\end{tabular}

diagnoses. For patients with sepsis, $82.7 \%$ of initial antibiotic regimens were changed during admission, in contrast to 54.7 and $50.3 \%$ of regimens for SSTI and UTI patients, respectively.

De-escalation was the most frequent first modification of antibiotic regimens and in total, $56.4 \%$ of first modifications were de-escalations, across all diagnoses (Table 3). For patients whose therapy was modified, the mean day of change was 3.6 days with $95 \%$ CI $(3.5,3.8)$. The time from start of antibiotic therapy to first change varied from patients with sepsis where day 3.0 with $95 \%$ CI $(2.7,3.3)$ was the mean day of change to patients with LRTI where change occurred on day 4.0 with $95 \% \mathrm{CI}(3.7,4.2)$.

In total, $84.5 \%$ of patients received oral antibiotics during the course of treatment (Table 3). Time to oral treatment differed substantially between diagnoses, from 2.7 days, $95 \%$ CI $(2.3,3.1)$ for UTI's to 5.1 days, $95 \%$ CI (4.6, 5.5) for sepsis.

\section{Duration of antibiotic therapy}

The mean duration (in-house and post-discharge) of antibiotic therapy was 10.6 days, $95 \%$ CI $(10.3,10.9)$ (Table 3). Mean days of in-house and post-discharge therapy was similar across all diagnosis. Patients diagnosed with sepsis had the highest mean number of inhouse antibiotic days at 6.6 days, $95 \%$ CI $(6.1,7.1)$, while patients with SSTI had the highest mean days of therapy after discharge and total days of antibiotics with 7.1, 95\% CI $(6.4,7.7)$ and 12.5 days $95 \%$ CI $(11.6,13.4)$, respectively. After discharge, $77.4 \%$ of patients continued with antibiotic therapy. 
Table 3 Antibiotic (AB) prescribing in hospitals - process measures

\begin{tabular}{|c|c|c|c|c|c|c|}
\hline & $\begin{array}{l}\text { LRTI }(n=412) \\
n(\%)\end{array}$ & $\begin{array}{l}\text { COPD ex }(n=280) \\
n(\%)\end{array}$ & $\begin{array}{l}\text { Sepsis }(n=248) \\
\text { n (\%) }\end{array}$ & $\begin{array}{l}\text { SSTI }(n=150) \\
n(\%)\end{array}$ & $\begin{array}{l}\text { UTI }(n=145) \\
\text { n (\%) }\end{array}$ & $\begin{array}{l}\text { Total }(N=1235) \\
\mathrm{n}(\%)\end{array}$ \\
\hline \multicolumn{7}{|l|}{$A B$ initiated } \\
\hline Emergency room & $320(77.7)$ & $244(87.1)$ & $240(96.8)$ & $135(90.0)$ & $94(64.8)$ & $1033(83.6)$ \\
\hline Ward & $92(22.3)$ & $36(12.9)$ & $8(3.2)$ & $15(10.0)$ & $51(35.2)$ & $202(16.4)$ \\
\hline \multicolumn{7}{|l|}{ Adherence to guideline } \\
\hline Yes & $280(68.0)$ & $177(63.2)$ & $151(60.9)$ & $90(60.0)$ & $80(55.2)$ & $778(63.0)$ \\
\hline No & $132(32.0)$ & $103(36.8)$ & $97(39.1)$ & $60(40.0)$ & $65(44.8)$ & $457(37.0)$ \\
\hline \multicolumn{7}{|c|}{ Accuracy between indication for AB-treatment and discharge infection diagnoses ${ }^{a}$} \\
\hline Yes & $331(80.3)$ & $255(91.1)$ & $103(41.5)$ & $143(95.3)$ & $122(84.1)$ & $954(77.3)$ \\
\hline No & $81(19.7)$ & $25(8.9)$ & $145(58.5)$ & $7(4.7)$ & $23(15.9)$ & $281(22.8)$ \\
\hline \multicolumn{7}{|l|}{ Empirical AB regimen was } \\
\hline Changed during admission & $232(56.3)$ & $167(59.6)$ & $205(82.7)$ & $82(54.7)$ & $73(50.3)$ & $759(61.4)$ \\
\hline Changed at discharge & $99(24.0)$ & $57(20.4)$ & $22(8.9)$ & $57(38.0)$ & $19(13.1)$ & $254(20.6)$ \\
\hline Continued at discharge & $32(7.8)$ & $29(10.4)$ & $2(0.8)$ & $5(3.3)$ & $34(23.5)$ & $102(8.3)$ \\
\hline Stopped & $49(11.9)$ & $27(9.6)$ & $19(7.7)$ & $6(4.0)$ & $19(13.1)$ & $120(9.7)$ \\
\hline \multicolumn{7}{|l|}{ Empirical $A B$ regimen was } \\
\hline De-escalated & $227(55.1)$ & $176(62.9)$ & $142(57.3)$ & $95(63.3)$ & $56(38.6)$ & $696(56.4)$ \\
\hline Escalated & $84(20.4)$ & $45(16.1)$ & $43(17.3)$ & $29(19.3)$ & $24(16.5)$ & $225(18.2)$ \\
\hline Changed-equal spectrum & $19(4.6)$ & $3(1.1)$ & $41(16.5)$ & $15(10.0)$ & $12(8.3)$ & $90(7.3)$ \\
\hline Unchanged $^{b}$ & $82(19.9)$ & $56(20.0)$ & $22(8.9)$ & $11(7.3)$ & $53(36.6)$ & $224(18.1)$ \\
\hline \multicolumn{7}{|c|}{ Time to change of first AB regimen $(n=1011)$} \\
\hline Mean $(95 \% \mathrm{Cl})^{\mathrm{C}}$ & $4.0(3.7,4.2)$ & $3.9(3.7,4.1)$ & $3.0(2.7,3.3)$ & $3.4(3.1,3.8)$ & $3.5(3.1,3.9)$ & $3.6(3.5,3.8)$ \\
\hline \multicolumn{7}{|c|}{ Number of treatment regimens through admission } \\
\hline 1 & $41(9.9)$ & $33(11.8)$ & $5(2.0)$ & $6(4.0)$ & $34(23.5)$ & $119(9.6)$ \\
\hline 2 & $245(59.5)$ & $187(66.8)$ & $86(34.7)$ & $77(51.3)$ & $75(51.7)$ & $670(54.3)$ \\
\hline 3 & $92(22.3)$ & $43(15.4)$ & $122(49.2)$ & $44(29.3)$ & $29(20.0)$ & $330(26.7)$ \\
\hline$>3$ & $34(8.3)$ & $17(6.1)$ & $35(14.1)$ & $23(15.3)$ & $7(4.8)$ & $116(9.4)$ \\
\hline \multicolumn{7}{|l|}{ Oral $A B$ given } \\
\hline Yes & $326(79.1)$ & $243(86.8)$ & $204(82.3)$ & $136(90.7)$ & $135(93.1)$ & $1044(84.5)$ \\
\hline No & $86(20.9)$ & $37(13.2)$ & $44(17.7)$ & $14(9.3)$ & $10(6.9)$ & $191(15.5)$ \\
\hline Mean first day $(95 \% \mathrm{Cl})$ & $4.2(3.9,4.5)$ & $3.6(3.3,3.8)$ & $5.1(4.6,5.5)$ & $4.8(4.3,5.3)$ & $2.7(2.3,3.1)$ & $4.1(3.9,4.3)$ \\
\hline \multicolumn{7}{|l|}{ First change of $A B$ regimen } \\
\hline During admission & $232(56.3)$ & $167(59.6)$ & $205(82.7)$ & $82(54.7)$ & $73(50.3)$ & $759(61.5)$ \\
\hline At discharge & $99(24.0)$ & $57(20.4)$ & $22(8.9)$ & $57(38.0)$ & $19(13.1)$ & $254(20.6)$ \\
\hline Continued at discharge & $32(7.8)$ & $29(10.4)$ & $2(0.8)$ & $5(3.3)$ & $34(23.5)$ & $102(8.3)$ \\
\hline Stopped & $49(11.9)$ & $27(9.6)$ & $19(7.7)$ & $6(4.0)$ & $19(13.1)$ & $120(9.7)$ \\
\hline \multicolumn{7}{|c|}{ Antibiotics prescribed at discharge } \\
\hline Yes & $296(71.8)$ & $214(76.4)$ & $193(77.8)$ & $139(92.7)$ & $114(78.6)$ & $956(77.4)$ \\
\hline No & $116(28.2)$ & $66(23.6)$ & $55(22.2)$ & $11(7.3)$ & $31(21.4)$ & $279(22.6)$ \\
\hline \multicolumn{7}{|l|}{ Days of $A B$ treatment } \\
\hline Mean $(95 \% \mathrm{Cl})^{\mathrm{d}}$ & $10.2(9.7,10.6)$ & $10.0(9.6,10.5)$ & $11.5(10.8,12.2)$ & $12.5(11.6,13.4)$ & $9.3(8.6,10.1)$ & $10.6(10.3,10.9)$ \\
\hline In-hospital & $6.3(5.9,6.7)$ & $6.0(5.7,6.4)$ & $6.6(6.1,7.1)$ & $6.0(5.3,6.6)$ & $5.3(4.8,5.8)$ & $6.2(5.9,6.4)$ \\
\hline After discharge $\mathrm{e}^{\mathrm{e}}$ & $5.5(5.2,5.8)$ & $5.2(4.9,5.6)$ & $6.3(5.8,6.8)$ & $7.1(6.4,7.7)$ & $4.9(4.5,5.3)$ & $5.8(5.6,6.0)$ \\
\hline
\end{tabular}

\footnotetext{
${ }^{a}$ Measured as match between initial grouped indication for treatment and grouped discharge diagnosis

b "Unchanged" includes patients where discontinuation of antibiotics was the only change

c Does not include patients who did not change initial antibiotic regimen (stop was only change)

${ }^{d}$ Does not include 40 patients where lenght of prescription treatment after discharge not stated in the discharge letter

${ }^{\mathrm{e}}$ Does not include 40 patients where length of prescription treatment was not stated in the discharge letter and 5 patients where length of prescriptions treatment was longer than 30 days
} 


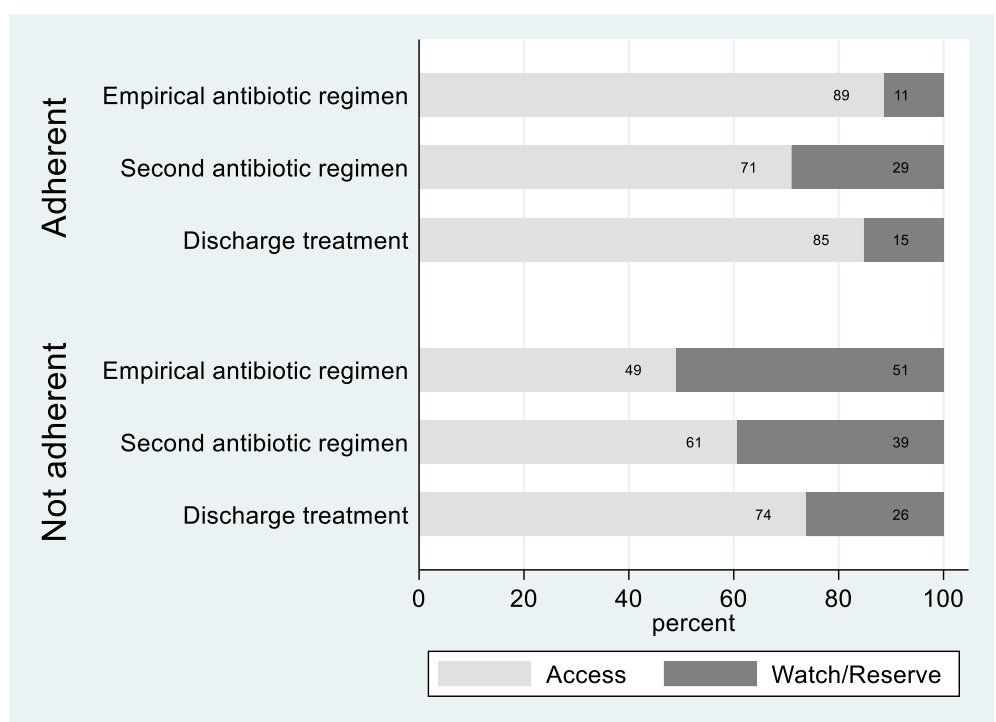

Fig. 1 Antibiotic regimens prescribed from admission to discharge, by AWaRe categories and adherence to guideline on initiation of therapy

\section{Discussion}

This study has identified key gaps and potential targets in the antibiotic prescribing process in hospitals for antibiotic stewardship interventions (Table 5).

One of the main aims of antibiotic stewardship programs is to reduce unnecessary use of broad-spectrum antibiotics. We applied WHO AWaRe categories to describe the categories of antibiotics prescribed and found that when initial antibiotic treatment were according to Norwegian national guidelines, the majority of regimens $(89 \%)$ consisted of only access group antibiotics. Nonadherent empirical regimens however, included several antibiotics from the watch/reserve category, but these regimens were often switched to regimens within the access categories upon first modification of treatment. At discharge, a greater number of regimens were from the access category, both suggestive of clinical microsystems that tried to adhere to guidelines and antibiotic stewardship principles, but also likely related to the restricted availability of oral broad-spectrum antibiotics in Norway.

In an American study from 2014, Braykov et al. ranked antibiotics in categories of narrow-spectrum, broadspectum, extended spectrum and restricted antibiotics [16]. Although there are some differences between the studies regarding the categories used to classify antibiotics, the results show that the prescription pattern is very different between the hospitals in the two studies. While $74 \%$ of patients in our study initially received antibiotics belonging only to the access group, most patients $(78 \%)$ had broad-spectrum and extended spectrum antibiotics prescribed as empirical therapy in the Braykov-study. This reflects the nature of the Norwegian national antibiotic guidelines, which mainly have antibiotics from the access group as first-line empirical treatment recommendations.

Initiating empirical antibiotic therapy is a crucial step in the treatment of infections and an important target for antibiotic stewardship interventions, as recently outlined by Tamma et al. in their paper describing the four moments of antibiotic decision making [17]. In our study, antibiotics were mainly prescribed in the emergency departments. The physicians responsible for prescribing are usually interns and residents and in Norwegian hospitals, junior doctors rely heavily on guidelines for antibiotic prescribing [18]. From a separate study by Skodvin et al., including patients from the same intervention study cohort, we also know that mean compliance with guidelines recommendations for microbiology testing practices was 89\% [19]. Most patients (83.6\%) started antibiotic treatment in the emergency departments and non-adherence to guidelines was higher when treatment was initiated at the wards, compared to the emergency departments $(\mathrm{OR}=1.7,95 \% \mathrm{CI}$ (1.24-2.36), $p=0.001)$. Other studies report reluctance from other medical teams to change therapy further down the line and together this highlights the need to focus on first-line clinical staff when planning antibiotic stewardship interventions [20].

Non-adherence to guidelines was also associated with hospital site and whether patients were admitted from an institution or not. Patients admitted from nursing homes or other institutions may have had treatment prior to hospital admission. A combination of age, frailty, comorbidity and increased fear of resistant bacteria as cause of infection could have caused physicians 
Table 4 Factors associated with non-adherence to antibiotic guideline

\begin{tabular}{|c|c|c|c|c|c|c|}
\hline & $\begin{array}{l}\text { Adherence }(n=778) \\
\mathrm{n}(\%)\end{array}$ & $\begin{array}{l}\text { Non-adherence } \\
(n=457) n(\%)\end{array}$ & $\begin{array}{l}\text { Univariate analysis } \\
\text { OR }(95 \% \mathrm{Cl})\end{array}$ & $p$-value & $\begin{array}{l}\text { Adjusted analysis }{ }^{a} \\
\text { OR }(95 \% \mathrm{Cl})\end{array}$ & $p$-value \\
\hline \multicolumn{7}{|l|}{$A B$ initiated } \\
\hline Emergency room & $670(64.9)$ & $363(35.1)$ & 1 & & 1 & \\
\hline Ward & $108(53.5)$ & $94(46.5)$ & $1.6(1.18,2.18)$ & 0.002 & $1.7(1.24,2.36)$ & 0.001 \\
\hline \multicolumn{7}{|c|}{ Indication for treatment } \\
\hline LRTI & $280(68.0)$ & $132(32.0)$ & 1 & & 1 & \\
\hline COPD ex & $177(63.2)$ & $103(36.8)$ & $1.23(0.90,1.70)$ & 0.196 & $1.42(1.03,1.98)$ & 0.035 \\
\hline Sepsis & $151(60.9)$ & $97(39.1)$ & $1.36(0.98,1.89)$ & 0.065 & $1.44(1.02,2.02)$ & 0.037 \\
\hline SSTI & $90(60.0)$ & $60(40.0)$ & $1.41(0.96,2.10)$ & 0.079 & $1.56(1.05,2.31)$ & 0.028 \\
\hline UTI & $80(55.2)$ & $65(44.8)$ & $1.72(1.17,2.54)$ & 0.006 & $1.62(1.09,2.41)$ & 0.017 \\
\hline \multicolumn{7}{|l|}{ Hospital } \\
\hline Hospital A & $376(60.8)$ & $242(39.2)$ & 1 & & 1 & \\
\hline Hospital B & $203(70.7)$ & $84(29.3)$ & $0.64(0.48,0.87)$ & 0.004 & $0.63(0.46,0.86)$ & 0.004 \\
\hline Hospital C & $199(60.3)$ & $131(39.7)$ & $1.02(0.78,1.34)$ & 0.872 & $0.95(0.71,1.26)$ & 0.712 \\
\hline \multicolumn{7}{|c|}{ Admitted from institution } \\
\hline No & $678(64.3)$ & $377(35.7)$ & 1 & & & \\
\hline Yes & $100(55.6)$ & $80(44.4)$ & $1.44(1.04,1.98)$ & 0.026 & $1.44(1.04,2.00)$ & 0.029 \\
\hline \multicolumn{7}{|c|}{ Accuracy between indication for $A B$-treatment and discharge infection diagnoses ${ }^{b}$} \\
\hline Yes & $604(63.3)$ & $350(36.7)$ & 1 & & 1 & \\
\hline No & $174(61.9)$ & $107(38.1)$ & $1.06(0.81,1.40)$ & 0.671 & $0.99(0.72,1.35)$ & 0.936 \\
\hline \multicolumn{7}{|l|}{ Sex } \\
\hline Male & $411(62.7)$ & $245(37.3)$ & 1 & & 1 & \\
\hline Female & $367(63.4)$ & $212(36.6)$ & $0.97(0.77 .1 .22)$ & 0.790 & $0.98(0.77,1.24)$ & 0.857 \\
\hline \multicolumn{7}{|l|}{ Age } \\
\hline$<45$ & $106(64.6)$ & $58(35.4)$ & 1 & & 1 & \\
\hline $46-65$ & $168(60.7)$ & $109(39.3)$ & $1.19(0.79,1.77)$ & 0.405 & $1.26(0.83,1.91)$ & 0.277 \\
\hline $66-85$ & $367(62.7)$ & $218(37.3)$ & $1.09(0.76,1.56)$ & 0.656 & $1.08(0.73,1.60)$ & 0.695 \\
\hline$>85$ & $137(65.6)$ & $72(34.4)$ & $0.96(0.63,1.47)$ & 0.854 & $0.87(0.54,1.37)$ & 0.540 \\
\hline \multicolumn{7}{|c|}{ Charlson Comorbidity Index } \\
\hline $\mathrm{CCl}=0$ & $304(65.8)$ & $158(34.2)$ & 1 & & 1 & \\
\hline $\mathrm{CCl}=1$ & $265(63.1)$ & $155(36.9)$ & $1.13(0.85,1.48)$ & 0.402 & $1.14(0.83,1.56)$ & 0.421 \\
\hline $\mathrm{CCl}=2$ & $102(57.9)$ & $74(42.1)$ & $1.40(0.98,1.99)$ & 0.066 & $1.35(0.93,1.97)$ & 0.115 \\
\hline $\mathrm{CCl}=3$ & $48(60.8)$ & $31(39.2)$ & $1.24(0.76,2.10)$ & 0.386 & $1.20(0.72,2.02)$ & 0.482 \\
\hline $\mathrm{CCl}=4$ & $26(61.9)$ & $16(38.1)$ & $1.18(0.62,2.27)$ & 0.611 & $1.18(0.60,2.33)$ & 0.626 \\
\hline $\mathrm{CCl}>4$ & $33(58.9)$ & $23(41.1)$ & $1.34(0.76,2.36)$ & 0.310 & $1.39(0.77,2.51)$ & 0.279 \\
\hline \multicolumn{7}{|c|}{ Number of working diagnoses } \\
\hline 1 & $493(64.6)$ & $270(35.4)$ & 1 & & 1 & \\
\hline 2 & $238(60.7)$ & $154(39.3)$ & $1.18(0.92,1.52)$ & 0.193 & $1.12(0.87,1.46)$ & 0.381 \\
\hline 3 & $47(58.8)$ & $33(41.2)$ & $1.28(0.80,2.05)$ & 0.299 & $1.18(0.73,1.92)$ & 0.491 \\
\hline \multicolumn{7}{|l|}{ Antibiotic allergies $^{c}$} \\
\hline No & $708(63.7)$ & $403(36.3)$ & 1 & & 1 & \\
\hline Yes & $69(56.6)$ & $53(43.4)$ & $1.35(0.92,1.97)$ & 0.120 & $1.40(0.95,2.06)$ & 0.088 \\
\hline \multicolumn{7}{|l|}{ eGFR } \\
\hline eGFR > 50 & $598(62.6)$ & $357(37.4)$ & 1 & & 1 & \\
\hline eGFR $<50$ & $180(64.3)$ & $100(35.7)$ & $0.93(0.71,1.23)$ & 0.611 & $0.88(0.66,1.17)$ & 0.378 \\
\hline
\end{tabular}

${ }^{a}$ All factors are adjusted for where $A B$ was initiated, indication for $A B$ treatment, hospital and admittance from institution

${ }^{b}$ Measured as match between initial grouped indication for treatment and grouped discharge diagnosis

c1 data missing 
Table $\mathbf{5}$ Identified gaps and potential targets for antibiotic stewardship interventions

\begin{tabular}{ll}
\hline Gaps identified & Potential targets \\
\hline $\begin{array}{l}\text { Guideline adherence increased the use of narrow spectrum WHO } \\
\text { Access group antibiotics in this study setting }\end{array}$ & $\begin{array}{l}\text { Promoting adherence to guidelines when prescribing empirical antibiotic } \\
\text { therapy }\end{array}$ \\
$\begin{array}{l}\text { Antibiotic therapy was initiated in the emergency room for } 83.6 \% \text { of } \\
\text { patients }\end{array}$ & $\begin{array}{l}\text { Targeting antibiotic prescribing in the emergency room, focusing on first } \\
\text { line clinical staff }\end{array}$ \\
$\begin{array}{l}\text { Non-adherence to antibiotic guideline was associated with admittance } \\
\text { from another institution }\end{array}$ & $\begin{array}{l}\text { Understanding the drivers for non-adherence in patients admitted from } \\
\text { institutions and focusing on antibiotic prescribing for this group of } \\
\text { patients }\end{array}$ \\
$\begin{array}{l}\text { Non-adherence to antibiotic guideline was associated with the place of } \\
\text { initiation of therapy, both regarding hospital site and wards compared } \\
\text { to emergency room }\end{array}$ & $\begin{array}{l}\text { Understanding the cultural and contextual drivers for antibiotic } \\
\text { prescribing across institutions and specialties }\end{array}$ \\
$\begin{array}{l}\text { Mean length of antibiotic therapy was similar across very different groups } \\
\text { of diagnosis. }\end{array}$ & $\begin{array}{l}\text { Focusing on reducing the duration of antibiotic therapy safely, in } \\
\text { accordance with emerging evidence on duration of antibiotic treatment }\end{array}$ \\
$\begin{array}{l}\text { Antibiotics prescribed upon discharge contributed significantly to the } \\
\text { total days of antibiotic therapy and the appropriateness of this practice } \\
\text { is often not clear }\end{array}$ &
\end{tabular}

to prescribe more broad-spectrum antibiotics for these patients. There is however, a need for a more thorough understanding of prescribing practices in this particular group of patients. Studies show that organisational culture influence antibiotic prescribing [20-22]. This could potentially explain why the odds ratio for non-adherent prescribing was significantly lower at hospital B than the two other included hospitals ( $\mathrm{OR}=0.6395 \% \mathrm{CI}(0.46$, $0.86), p=0.004)$. It also signals that a thorough understanding of organisational culture with barriers and facilitators for prudent antibiotic prescribing is an important part of planning for antibiotic stewardship interventions.

Empirical antibiotic regimens were usually modified during admission (61.5\%) and oral antibiotics were prescribed for $84.5 \%$ of patients. Other studies looking at the process of antibiotic prescribing in hospitals have focused on review of empirical therapy in relation to patient outcome or effect of interventions on prescribing process measures [23-25]. Braykov et al. found that by the 5th day of therapy, $21,5 \%$ of empirical antibiotics were narrowed or discontinued, while Aillet et al. found that antibiotic review was performed in $69 \%$ of patients with bacteraemia $[16,24]$. In comparison, although we did not measure all patients at one specific day, $74,5 \%$ of empirical antibiotics were de-escalated (56,4\%) and stopped $(18,1 \%)$ as first modification of therapy in our study. Modifications happened between day 3 and 4 when initiation of therapy was defined as day 1 . This is in agreement with recommendations stating that review of therapy should take place $48-72 \mathrm{~h}$ after initiation of antibiotic therapy [26-28]. Upon discharge, $77.4 \%$ of patients continued antibiotic treatment and the mean length of post-discharge therapy was similar to the mean length of in-house treatment. This could mean either that most patients were not fully recovered upon discharge or that antibiotics were continued "just in case," justifying an earlier discharge and giving the physician reassurance for the patients' well-being. The lack of documentation regarding length of antibiotic therapy has been heavily debated and studies suggest shorter antibiotic courses are safe and effective for an increasing number of diagnoses [29-33]. In our study, there was a remarkable similarity in duration of antibiotic therapy between the various groups of diagnoses, both inhospital and post-discharge. For all patients, the mean number of days of antibiotic therapy were 10.6 days and the range for the various groups were narrow (9.3 to 12.5 days) when post-discharge therapy was included. There is a need for more studies, informing policymakers and clinicians about the optimal duration of antibiotic therapy for individual diagnosis, both inhospital and for post-discharge use.

This study has some limitations. When assessing adherence to guidelines on initiation of treatment, we used the indication for treatment stated in the electronic medical record, and this was usually a working diagnosis on admission. The diagnosis may change with more data and results available. To check whether this constituted a major issue for interpretation of data, we looked at the coherence between indication for treatment and the infection discharge diagnosis (if present) in the discharge letter. The group of diagnoses for which this might be an issue, is sepsis, where accuracy between indication for treatment and discharge diagnosis was low. During the study period, SIRS-criteria were used to screen patients for sepsis. SIRS identify more patients with suspected sepsis than the qSOFA score, which is currently in use. The low accuracy in this group could be due to lack of documentation of sepsis at discharge, with only the original focus of the infection often documented in discharge papers. It is possible that review of therapy took place without modifications to the patient's antibiotic regimen. Such reviews were not identified during data collection and represents a limitation to this study. We also did not take 
dosing into consideration when assessing adherence to guidelines and modification of therapy. Appropriateness of antibiotic therapy was not evaluated after initial assessment of adherence to guidelines for empirical antibiotic treatment. It is therefore unknown whether escalation, deescalation, stop or change was the best option for each individual patient. From the study by Skodvin et al., with patients derived from the same intervention study cohort, we do however know that only $18 \%$ of patients had applicable microbiology test results and for only half of these patients ( $9 \%$ of the total cohort), these findings were used to guide therapy [19].

The Nordic countries and the Netherlands are currently in a favourable position regarding antimicrobial resistance and are still able to utilize the most ecologically friendly antibiotics in empirical regimens. Exploring different ways of aggregating and analysing data to understand hospital antibiotic prescribing processes are however important in all countries and institutions, aiming to identify targets for stewardship interventions.

Future studies should include assessment of appropriateness of therapy throughout the hospital stay to have a more comprehensive review of prescribing quality at every step of the process. Identifying and studying contributions from other healthcare professionals, like nurses and pharmacist and the team effort in antibiotic stewardship would also be valuable. As patient involvement and empowerment is increasing, the contribution of patients in antibiotic stewardship in hospital settings should also be investigated. Such studies could contribute to the identification of more targets for antibiotic stewardship interventions.

\section{Conclusions}

Analysis of patient level antibiotic prescribing data and the use of WHO AWaRe to categorise antibiotic regimens throughout the hospital stay, identified relevant targets for antibiotic stewardship interventions in our population of hospital inpatients. Identified targets included 1) adherence to guidelines 2) focus on prescribing physicians in the emergency room 3) understanding prescribing for patients admitted from an institution 4) organisational culture and 5) duration of antibiotic therapy.

\section{Supplementary information}

Supplementary information accompanies this paper at https://doi.org/10. 1186/s13756-020-00749-y.

Additional file 1: Table 1. Grouping of indication for treatment. Table 2. Overview of AWaRe categories with study modifications. Table $\mathbf{3}$. Evaluation of antimicrobial spectrum and categorization of change.

\section{Abbreviations}

AB: Antibiotic; AWaRe: Access, Watch, Reserve; CCl: Charlson Comorbidity Index; COPD ex: Exacerbation of Chronic Obstructive Pulmonary Disease;
eGFR: Estimated Glomerular Filtration Rate; UTI: Urinary Tract Infection; LOS: Length of stay; LRTI: Lower Respiratory Tract Infection; SIRS: Systemic Inflammatory Response Syndrome; SSTI: Skin- and Soft Tissue Infections; qSOFA: Quick Sequential [Sepsis-related] Organ Failure Assessment score; WHO: World Health Organization

\section{Acknowledgements}

We would like to thank all included hospitals for contributing data to this study and Marion Iren Neteland and Torhild Vedeler for contributing to data collection.

\section{Authors' contributions}

JSW, BS and IS designed the study and data was collected by JSW, IS, LKSK, BS, MIN and TV. JSW, IS, LKSK and BS validated data and JSW, RMN, BS and IS did the data analysis. All authors performed data interpretation, while JSW, BS, ES and IS wrote the manuscript. All authors critically assessed the manuscript throughout the writing process and also read and approved the final version.

\section{Funding}

This work was internally funded by Haukeland University Hospital, The Hospital Pharmacies Enterprise in Western Norway and Stavanger University Hospital for collection, validation and analysis of data and preparation of the manuscript for authors JSW, BS, IS, SH, RMN and LKSK. The work of EC was funded by the National Institute for Health Research, UK Department of Health (HPRU-2012-10047), in partnership with Public Health England and the Economic and Social Sciences Research Council (ESRC). The funding bodies had no role in the design of the study, in collection, analysis and interpretation of data or in writing of the manuscript.

\section{Availability of data and materials}

The datasets generated and/or analysed during the current study are not publicly available in concordance with the approval from the Data Protection Officer (2013/9352), but are available from the corresponding author on reasonable request.

\section{Ethics approval and consent to participate}

The study was performed in accordance with the Declaration of Helsinki [34] The study was approved by the local data protection officer (2013/9352) and the regional ethical committee of Western Norway approved the waiver of informed consent (2013/1305). All patients treated with antibiotics received an information leaflet at the ward about the study and provided the opportunity to withdraw from the study.

Consent for publication

Not applicable.

\section{Competing interests}

The authors declare that they have no competing interests.

\section{Author details}

${ }^{1}$ Department of Clinical Science, University of Bergen, Jonas Lies vei 87, 5021 Bergen, Norway. ${ }^{2}$ Norwegian Advisory Unit for Antibiotic Use in Hospitals, Department of Research and Development, Haukeland University Hospital, Jonas Lies vei 65, 5021 Bergen, Norway. ${ }^{3}$ Department of Quality and Development, Hospital Pharmacies Enterprise in Western Norway, Møllendalsbakken 9, 5021 Bergen, Norway. ${ }^{4} \mathrm{NHIR}$ Health Protection Research Unit in Healthcare Associated Infections and Antimicrobial Resistance, Imperial College London, Hammersmith Hospital Campus, Du Cane Road, London W12 ONN, UK. ${ }^{5}$ Department of Drug Statistics, Norwegian Institute of Public Health, Marcus Thranes gate 6, 0473 Oslo, Norway. ${ }^{6}$ School of Pharmacy, University of Oslo, Sem Sælandsvei 3, 0371 Oslo, Norway. ${ }^{7}$ Western Norway University of Applied Sciences, Inndalsveien 28, 5063 Bergen, Norway. ${ }^{8}$ Department of Infectious Diseases and Unit for Infection Prevention and Control, Department of Research and Education, Stavanger University Hospital, Armauer Hansens vei 20, 4011 Stavanger, Norway. ${ }^{9}$ Department of Pharmaceutical Services, Oslo Hospital Pharmacy, Kirkeveien 166, 0450 Oslo, Norway. ${ }^{10}$ Innovation, Access and Use, Department of Essential Medicines and Health Products, World Health Organization (WHO), Avenue Appia 20, 1211 Geneva 27, Switzerland. 
Received: 16 August 2019 Accepted: 4 June 2020

Published online: 21 July 2020

\section{References}

1. Bronzwaer SL, Cars O, Buchholz U, Molstad S, Goettsch W, Veldhuijzen IK, et al. A European study on the relationship between antimicrobial use and antimicrobial resistance. Emerg Infect Dis. 2002;8(3):278-82.

2. European Centre for Disease Prevention and Control. Antimicrobial consumption. Annual Epidemiological Report for 2017. Stockholm: ECDC 2018. https://ecdc.europa.eu/sites/portal/files/documents/AER_for_2017antimicrobial-consumption.pdf. Accessed 20 Apr 2019.

3. Haug JB, Reikvam A. WHO defined daily doses versus hospital-adjusted defined daily doses: impact on results of antibiotic use surveillance. J Antimicrob Chemother. 2013;68(12):2940-7.

4. NORM/NORM-VET. Usage of Antimicrobial Agents and Occurrence of Antimicrobial Resistance in Norway 2017. Tromso/Oslo: Norwegian Surveillance System for Antibiotic Resistance in Microbes (NORM), Norwegian Veterinary Institute, Norwegian Institute of Public Health; 2018. https://unn.no/Documents/Kompetansetjenester,\%20-sentre\%20og\%2 ofagråd/NORM\%20-\%20Norsk\%20overvåkingssystem\%20for\%2 Oantibiotikaresistens\%20hos\%20mikrober/Rapporter/NORM_NORM-VET_201 7.pdf.. Accessed 26 Sep 2018.

5. Bitterman R, Hussein K, Leibovici L, Carmeli Y, Paul M. Systematic review of antibiotic consumption in acute care hospitals. Clin Microbiol Infect. 2016; 22(6):561.e7-e19.

6. Davey P, Marwick CA, Scott CL, Charani E, McNeil K, Brown E, et al. Interventions to improve antibiotic prescribing practices for hospital inpatients. Cochrane Database of Systematic Reviews. 2017. https://doi.org/ 10.1002/14651858.CD003543.pub4:(2.

7. Aldeyab MA, Kearney MP, McElnay JC, Magee FA, Conlon G, Gill D, et al. A point prevalence survey of antibiotic prescriptions: benchmarking and patterns of use. Br J Clin Pharmacol. 2011;71(2):293-6.

8. Howard P, Huttner B, Beovic B, Beraud G, Kofteridis DP, Pano Pardo J, et al. ESGAP inventory of target indicators assessing antibiotic prescriptions: a cross-sectional survey. J Antimicrob Chemother. 2017;72(10):2910-4.

9. Sharland M, Pulcini C, Harbarth S, Zeng M, Gandra S, Mathur S, et al. Classifying antibiotics in the WHO essential medicines list for optimal use-be AWaRe. Lancet Infect Dis. 2018;18(1):18-20.

10. Hsia Y, Sharland M, Jackson C, Wong ICK, Magrini N, Bielicki JA. Consumption of oral antibiotic formulations for young children according to the WHO access, watch, reserve (AWaRe) antibiotic groups: an analysis of sales data from 70 middle-income and high-income countries. Lancet Infect Dis. 2019;19(1):67-75.

11. Wathne JS, Kleppe LKS, Harthug S, Blix HS, Nilsen RM, Charani E, et al. The effect of antibiotic stewardship interventions with stakeholder involvement in hospital settings: a multicentre, cluster randomized controlled intervention study. Antimicrobial Resistance Infection Control. 2018;7(1):109.

12. Norwegian Directorate of Health. Norwegian National Clinical Guideline for Antibiotic Use in Hospitals. 2013. https://helsedirektoratet.no/retningslinjer/ antibiotika-i-sykehus. Accessed 3 Jan 2016.

13. Charlson ME, Pompei $P$, Ales KL, MacKenzie CR. A new method of classifying prognostic comorbidity in longitudinal studies: development and validation. J Chronic Dis. 1987;40(5):373-83.

14. Stagg V. Charlson: Stata module to calculate Charlson index of comorbidity. Orebro University School of Business 2017. https://econpapers.repec.org/ software/bocbocode/s456719.htm. Accessed 06 Dec 2018.

15. World Health Organization (WHO). WHO Model List of Essential Medicines. 2017. http://www.who.int/medicines/publications/essentialmedicines/20th_ EML2017_FINAL_amendedAug2017.pdf?ua=1. Accessed 2 Aug 2018.

16. Braykov NP, Morgan DJ, Schweizer ML, Uslan DZ, Kelesidis T, Weisenberg SA, et al. Assessment of empirical antibiotic therapy optimisation in six hospitals: an observational cohort study. Lancet Infect Dis. 2014;14(12):1220-7.

17. Tamma PD, Miller MA, Cosgrove SE. Rethinking How Antibiotics Are Prescribed: Incorporating the 4 Moments of Antibiotic Decision Making Into Clinical Practice. JAMA. 2019;321(2):139-40.

18. Skodvin B, Aase K, Charani E, Holmes A, Smith I. An antimicrobial stewardship program initiative: a qualitative study on prescribing practices among hospital doctors. Antimicrob Resist Infect Control. 2015;4:24.

19. Skodvin B, Wathne JS, Lindemann PC, Harthug S, Nilsen RM, Charani E, et al. Use of microbiology tests in the era of increasing AMR rates- a multicentre hospital cohort study. Antimicrob Resist Infect Control. 2019;8:28.
20. Charani E, Ahmad R, Rawson TM, Castro-Sanchez E, Tarrant C, Holmes AH. The differences in antibiotic decision-making between acute surgical and acute medical teams: an ethnographic study of culture and team dynamics. Clin Infect Dis. 2019;69(1):12-20. https://doi.org/10.1093/cid/ciy844.

21. Ukawa N, Tanaka M, Morishima T, Imanaka Y. Organizational culture affecting quality of care: guideline adherence in perioperative antibiotic use. Int J Qual Health Care. 2015;27(1):37-45.

22. Charani E, Castro-Sanchez E, Sevdalis N, Kyratsis Y, Drumright L, Shah N, et al. Understanding the determinants of antimicrobial prescribing within hospitals: the role of "prescribing etiquette". Clin Infect Dis. 2013;57(2):188-96.

23. Viasus D, Simonetti AF, Garcia-Vidal C, Niubo J, Dorca J, Carratala J. Impact of antibiotic de-escalation on clinical outcomes in community-acquired pneumococcal pneumonia. J Antimicrob Chemother. 2017;72(2):547-53.

24. Aillet C, Jammes D, Fribourg A, Leotard S, Pellat O, Etienne P, et al. Bacteraemia in emergency departments: effective antibiotic reassessment is associated with a better outcome. Eur J Clin Microbiol Infect Dis. 2018;37(2): 325-31.

25. Lesprit P, Landelle C, Girou E, Brun-Buisson C. Reassessment of intravenous antibiotic therapy using a reminder or direct counselling. J Antimicrob Chemother. 2010;65(4):789-95.

26. Public Health England. Start Smart - Then Focus. Antimicrobial Stewardship Toolkit for English Hospitals. London: Public Health England. 2015. https:// assets.publishing.service.gov.uk/government/uploads/system/uploads/ attachment_data/file/417032/Start_Smart_Then_Focus_FINAL.PDF. Accessed 9 June 2019.

27. Centers for Disease Control and Prevention (CDC). Core Elements of Hospital Antibiotic Stewardship Programs. Atlanta: US Department of Health and Human Services, CDC. 2014. https://www.cdc.gov/antibiotic-use/ healthcare/pdfs/core-elements.pdf. Accessed 9 June 2019.

28. Norwegian Department of Health and Care Services. Action plan against antibiotic resistance in health services. Oslo; 2015. https://www.regjeringen. no/contentassets/915655269bc04a47928fce917e4b25f5/handlingsplanantibiotikaresistens.pdf. Accessed 21 Mar 2016.

29. Llewelyn MJ, Fitzpatrick JM, Darwin E, SarahTonkin-Crine GC, Paul J, et al. The antibiotic course has had its day. Bri Med J. 2017;358:j3418.

30. Spellberg B. The new antibiotic mantra- "shorter is better"editorial. JAMA Intern Med. 2016;176(9):1254-5.

31. Uranga A, Espana PP, Bilbao A, Quintana JM, Arriaga I, Intxausti M, et al. Duration of antibiotic treatment in community-acquired pneumonia: a multicenter randomized clinical trial. JAMA Intern Med. 2016;176(9):1257-65.

32. Yahav D, Franceschini E, Koppel F, Turjeman A, Babich T, Bitterman R, et al. Seven versus fourteen days of antibiotic therapy for uncomplicated gramnegative bacteremia: a non-inferiority randomized controlled trial. Clin Infect Dis. 2019;69(7):1091-8. https://doi.org/10.1093/cid/ciy1054.

33. Dawson-Hahn EE, Mickan S, Onakpoya I, Roberts N, Kronman M, Butler CC, et al. Short-course versus long-course oral antibiotic treatment for infections treated in outpatient settings: a review of systematic reviews. Fam Pract. 2017:34(5):511-9.

34. World Medical A. World medical association declaration of Helsinki: ethical principles for medical research involving human subjects. JAMA. 2013; 310(20):2191-4

\section{Publisher's Note}

Springer Nature remains neutral with regard to jurisdictional claims in published maps and institutional affiliations.

\section{Ready to submit your research? Choose BMC and benefit from:}

- fast, convenient online submission

- thorough peer review by experienced researchers in your field

- rapid publication on acceptance

- support for research data, including large and complex data types

- gold Open Access which fosters wider collaboration and increased citations

- maximum visibility for your research: over $100 \mathrm{M}$ website views per year

At BMC, research is always in progress.

Learn more biomedcentral.com/submission 